\title{
Slotted Aloha with multi-AP diversity and APS transmit beamforming
}

\author{
Di Zheng and Yu-Dong Yao*
}

\begin{abstract}
Slotted Aloha is an effective random access protocol and can also be an important element of more advanced media access protocols. This paper investigates slotted Aloha in a radio environment with multiple access points. Specifically, we examine the impact of multi-access-point (multi-AP) diversity on the performance of slotted Aloha. The paper considers both omni-directional (OM) and beamforming (BF) antennas at transmission nodes. This leads to the investigation and comparison of four different network scenarios, i.e., OM with multi-AP diversity, OM without multi-AP diversity, BF with multi-AP diversity and BF without multi-AP diversity. Performance evaluations and comparisons are presented in terms of throughput and average packet delay.
\end{abstract}

Keywords: Slotted Aloha, Multi-access-point diversity, Beamforming, Capture effect, Rayleigh fading, Throughput, Average packet delay

\section{Introduction}

Slotted Aloha has been extensively used in wireless environments [1-4], in which the power levels of received packets can be different due to independent fading. It is possible that the strongest packet captures the receiver even when there is a packet collision [5], which could increase throughput. This phenomenon is referred to as the capture effect. A lot of research have been conducted for the investigations of the capture effect under various fading channels, including Rayleigh, Rician and Nakagami [6-8].

Besides the capture effect, beamforming (BF) techniques can also potentially increase throughput since they are able to reduce collisions in slotted Aloha as compared to omni-directional (OM) antennas. The applications of $\mathrm{BF}$ at both receiving and transmitting sides have been investigated. It is shown that a single-beam adaptive array at the receiver improves the performance of a slotted Aloha network by creating a strong capture effect [9] and a multiple receiving beam adaptive array can successfully receive two or more overlapping packets at the same time [10]. Slotted Aloha using transmit $\mathrm{BF}$ at mobile entities in mobile ad hoc networks has also been studied [11].

\footnotetext{
* Correspondence: yyao@stevens.edu

Department of Electrical and Computer Engineering, Stevens Institute of Technology, Hoboken, NJ 07030, USA
}

Notice that there can be two types of interference in slotted Aloha in a cellular environment, multiple access interference and cochannel interference. For a given user, multiple access interference is due to users within the same cell and cochannel interference is due to users in cochannel cells. The performance of slotted Aloha in Nakagami fading channels considering both synchronized and asynchronous cochannel cells is analyzed in [12], highlighting the differences between these two types of interference. While all cochannel interfering packets are discarded in [12], a model, in which multiple base stations are able to accept a packet from the same user as long as it captures the receivers, is studied in [13] through simulations. Clearly, such a scheme potentially improves the throughput of slotted Aloha as compared to the approach in [12].

The model in [13] is a type of multi-access-point (multi-AP) diversity, a concept also addressed in [14] which considers downlinks in cellular communications. It is pointed out that a user can simultaneously receive pilot channels from multiple base stations, which introduces multi-AP diversity due to independent channel variations between the user and the base stations [14]. Therefore, a user could choose one base station among a set of base stations as its server according to channel conditions. Similarly, a multi-AP

\section{Springer}


architecture has been proposed for wireless local area networks, in which one user can associate with more than one access point [15].

This paper investigates slotted Aloha with multi-AP diversity and it differs from previous research in the following aspects. Firstly, we develop analytical models and derive closed-form solutions for the throughput and average packet delay. Secondly, we investigate the joint use of transmit BF and multi-AP diversity. We thus specifically study four network scenarios, i.e., OM with multi-AP diversity, OM without multi-AP diversity, BF with multi-AP diversity and BF without multi-AP diversity, to exam and compare various technical options.

The rest of this paper is organized as follows. Section 2 gives the system model of slotted Aloha with multiAP diversity, including two cases in which OM and directional antennas are applied, respectively. Sections 3 and 4 analyze these two cases and derive the capture probabilities, throughput and average packet delay. In Section 5, numerical results are presented and, finally, Section 6 draws conclusions.

\section{System Model}

\section{A. Network model}

We consider a network with two access points (AP) $A$ and $B$ (two servers) (Figure 1) placed to cover a given area. Around AP $A$, there are a set of $N_{A}$ users (User Set $A$ ), and around AP $B$, there are a set of $N_{B}$ users (User Set $B)$. A user $u_{i}\left(1 \leq i \leq N_{A}\right)$ in User Set $A$ transmits its packet to AP $A$ and/or AP $B$ depending on its antenna structures (OM or BF). Similarly, a user $v_{j}\left(1 \leq j \leq N_{B}\right)$ in User Set B transmits its packet to AP B and/or AP $A$.

We apply a traffic and retransmission model as in [16]. If no packet retransmission is needed, each user generates a new packet with a probability $\sigma$ and no packet with a probability $1-\sigma$ during each time slot. Once a user generates a packet, it transmits the packet immediately. If the packet transmission fails, it will be retransmitted in each of the following slots with a probability $\sigma$ until it is successfully transmitted. When a user needs to perform packet retransmissions, it does not generate any new packet.

\section{B. Signal capture model}

A transmission collision in fading channels does not always result in transmission failures of all packets due to the capture effect, in which a packet may capture a receiver if its power level is higher than the sum of powers of all interfering packets $[17,18]$. The capture probability, $P_{\text {cap }}$, can thus be calculated by

$$
P_{\text {cap }}(I, J)=\operatorname{Pr}\left(\frac{x}{\sum_{i=1}^{I} y_{i}+\sum_{j=1}^{J} z_{j}}>R\right)
$$

for $R \geq 1, I \geq 0, J \geq 0$, where $x$ is the power of the desired packet; $R$ is a capture ratio; $I$ and $J$ are the total numbers of interfering packets from the same user set as the desired packet and from the other user set, respectively. $y_{i}$ and $z_{j}$ indicate the powers of interfering packets from the two user sets. In a Rayleigh fading channel, $x, y_{i}, z_{j}$ follow exponential distributions $[17,19]$. There are two scenarios in determining the mean powers of $x, y_{i}$, and $z_{j}$. When the desired packet is transmitted from User Set $A$ (or $B$ ) to AP $A$ (or $B$ ), the mean powers are assumed to be $X, Y$ and $\mathcal{Z}$. When the desired packet is transmitted from User Set $A$ (or $B$ ) to AP $B$ (or $A$ ), the mean powers are assumed to be $\mathcal{X}, \mathcal{Y}$ and $Z$. Notice that the mean powers $X, Y$ and $Z$ relate to packet transmissions (desired or interfering) from User Set $A$ (or $B$ ) to AP $A$ (or $B$ ). The mean powers $\mathcal{X}$, $\mathcal{Y}$ and $\mathcal{Z}$ relate to packet transmissions (desired or interfering) from User Set $A$ (or $B$ ) to AP $B$ (or $A$ ). Figure 2 illustrates the packet transmissions and the notations of signal and interference powers and their mean powers. We assume that the mean powers satisfy

$$
X=Y=Z
$$

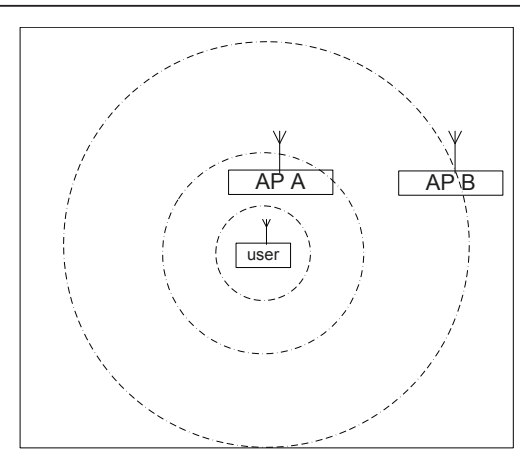

(a)

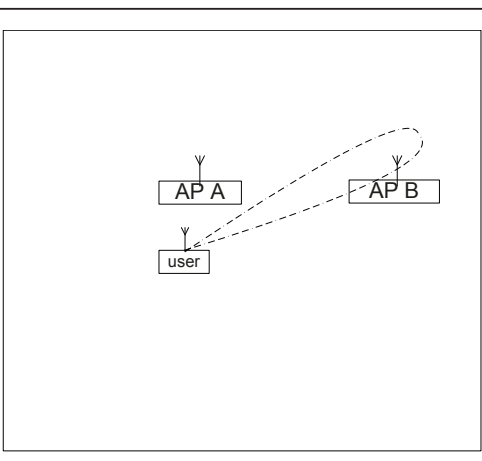

(b)

Figure 1 System model. (a) Omnidirectional antenna, (a) Beamforming antenna. 


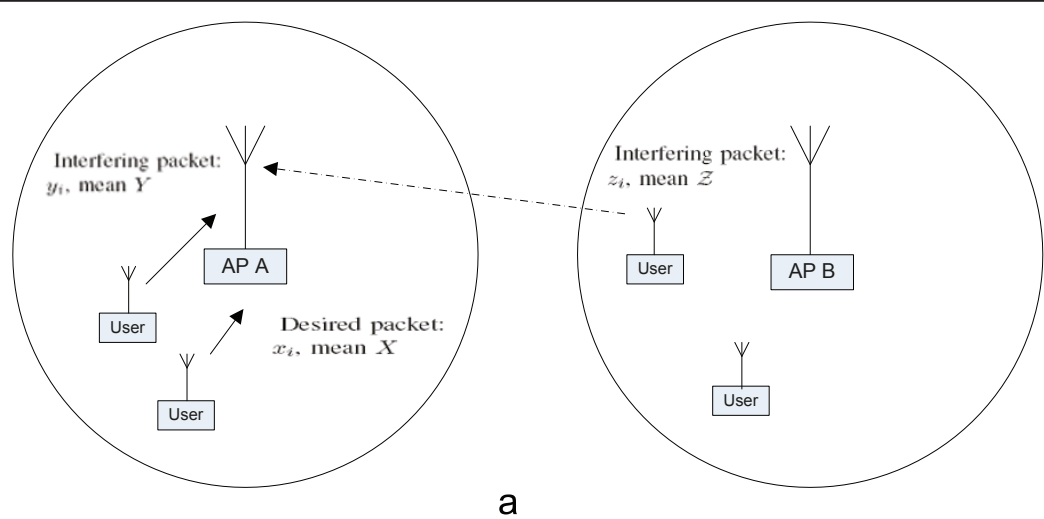

a

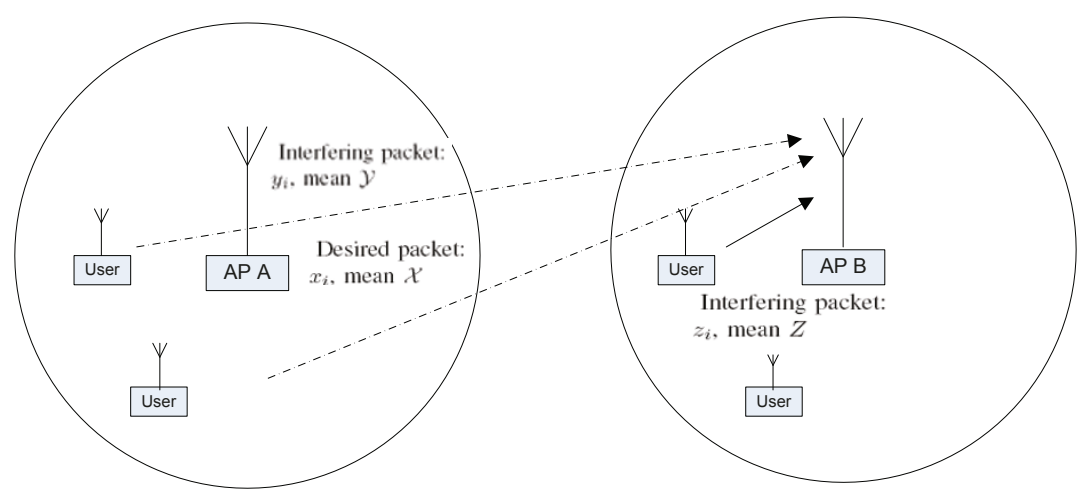

b

Figure 2 Signal and interference modeling

and

$$
\mathcal{X}=\mathcal{Y}=\mathcal{Z}
$$

We further define

$$
\gamma=\frac{\mathcal{X}}{X}=\frac{\mathcal{Y}}{Y}=\frac{\mathcal{Z}}{Z}
$$

Notice that the signal and capture model consider a Rayleigh fading channel environment. There are several capture models which have been investigated in literatures [17-20]. This paper only considers one model as defined in Equation 1. Near-far effects $[19,20]$ due to user spatial distributions are not considered in this model and the combined effect of Rayleigh fading and user spatial distributions will be investigated in our future research.

\section{Multi-AP diversity}

Multi-AP diversity, in which one user can be associated with more than one access point (e.g., base stations in cellular networks or hot spots in wireless local area networks), is investigated in $[14,15]$. In the network model we defined above, each user could potentially transmit a packet through two independent channels to two APs. Therefore, there is multi-AP diversity in the system to potentially provide diversity gains. The following explains how the diversity is exploited when $\mathrm{OM}$ or BF antennas are applied at the transmit side.

\section{OM versus $B F$ antennas}

When users employ OM transmit antennas, any packet transmitted by any user can potentially reach both APs (see Figure 1a). Therefore, a packet has to compete with other packets from all users (User Set A and User Set B) in order to capture a receiver. If transmit BF is used, each user can choose one AP as its server where its packet will have stronger power as compared to that at the other AP. Such an AP selection task can be accomplished based on feedback information or pilot signals. The user steers its beam towards only the chosen AP. Therefore, under the BF antenna mode, any packet can only reach one AP (see Figure $1 \mathrm{~b}$ ). And this leads to potentially less interference.

\section{Slotted Aloha with Multi-AP Diversity and OM Antenna}

A. Capture probability

Considering the transmission of a desired packet from User Set $A$ to AP A, following the definition in Section 
2.A, we find its capture probability as follows,

$$
\begin{aligned}
& P_{\mathrm{cap}_{A} \rightarrow A}(I, J)=\operatorname{Pr}\left(\frac{x}{\sum_{i=1}^{I} y_{i}+\sum_{j=1}^{I} z_{j}}>R\right) \\
& =\int_{0}^{\infty} \cdots \int_{0}^{\infty} \int_{R\left(\sum_{i=1}^{l} y_{t}+\sum_{j=1}^{l} z_{j}\right)} \frac{1}{X} e^{-\frac{x}{X}} d x \prod_{i=1}^{I} \frac{1}{Y} e^{-\frac{y_{Y}}{Y}} \prod_{j=1}^{I} \frac{1}{Z^{-\frac{z_{Y}}{Z}}} d y_{1} \cdots d y_{1} d z_{1} \cdots d z_{j}(5) \\
& =\left(\frac{X}{R Y+X}\right)^{I}\left(\frac{X}{R Z+X}\right)^{\prime}
\end{aligned}
$$

Following (2)-(4), (5) can be rewritten as

$$
P_{\mathrm{CapS}_{A} \rightarrow A}(I, J)=\left(\frac{1}{R+1}\right)^{I}\left(\frac{1}{R \gamma+1}\right)^{J}
$$

Similarly, considering other transmission scenarios, we are able to obtain the following capture probabilities (from User Set $A$ to AP B, from User Set B to AP B, and from User Set $B$ to AP $A$ ).

$$
\begin{aligned}
& P_{\mathrm{capS}_{A} \rightarrow B}(I, J)=\left(\frac{1}{R+1}\right)^{I}\left(\frac{\gamma}{R+\gamma}\right)^{J} \\
& P_{\mathrm{capS}_{B} \rightarrow B}(I, J)=\left(\frac{1}{R+1}\right)^{I}\left(\frac{1}{R \gamma+1}\right)^{J} \\
& P_{\mathrm{CapS}_{B} \rightarrow A}(I, J)=\left(\frac{1}{R+1}\right)^{I}\left(\frac{\gamma}{R+\gamma}\right)^{J}
\end{aligned}
$$

\section{B. Throughput}

We consider the throughput per AP, $S$, which is defined as the total number of packets successfully received by the two APs during one time slot and divided by two. The following defines several events during a period of one time slot.

E: AP $A$ successfully receives one packet and AP $B$ successfully receives one packet and the packets are different.

$F$ : AP $A$ and AP $B$ both successfully receive the same packet.

G: Only AP $A$ successfully receives a packet.

$H$ : Only AP $B$ successfully receives a packet.

$T_{i, j}$ : There are $i$ users in User Set $A$ and $j$ users in User Set $B$ attempting to transmit. If one packet is received successfully at both APs, it is only counted as one. The throughput is thus calculated as

$$
\begin{aligned}
S= & 0.5 \times[2 \operatorname{Pr}(E)+\operatorname{Pr}(F)+\operatorname{Pr}(G)+\operatorname{Pr}(H)] \\
= & 0.5 \times\left\{\sum_{i=0}^{N_{A}} \sum_{j=0}^{N_{B}}\left(\begin{array}{c}
N_{A} \\
i
\end{array}\right) \sigma^{i}(1-\sigma)^{N_{A}-i}\left(\begin{array}{c}
N_{B} \\
j
\end{array}\right) \sigma^{j}(1-\sigma)^{N_{B}-j}\right. \\
& \left.\times\left[2 \operatorname{Pr}\left(E \mid T_{i, j}\right)+\operatorname{Pr}\left(F \mid T_{i, j}\right)+\operatorname{Pr}\left(G \mid T_{i, j}\right)+\operatorname{Pr}\left(H \mid T_{i, j}\right)\right]\right\}
\end{aligned}
$$

in which

$$
\begin{aligned}
\operatorname{Pr}\left(E \mid T_{i, j}\right)= & \operatorname{Pr}\left(\operatorname{AP} A \text { successfully receives a packet } \mid T_{i, j}\right) \\
& \times \operatorname{Pr}\left(\mathrm{AP} B \text { successfully receives a packet } \mid T_{i, j}\right) \\
& -\left(\operatorname{Pr}\left(\text { A user in User Set } A \text { successfully transmits a packet to AP A and AP } B \mid T_{i, j}\right)\right. \\
& \left.+\operatorname{Pr}\left(\text { A user in User Set } B \text { successfully transmits a packet to AP A and AP } B \mid T_{i, j}\right)\right)
\end{aligned}
$$

where

$$
\begin{aligned}
& \operatorname{Pr}\left(\mathrm{AP} A \text { successfully receives a packet } \mid T_{i, j}\right) \\
= & i P_{\mathrm{cap}_{A} \rightarrow A}(i-1, j)+j P_{\mathrm{cap}_{B} \rightarrow A}(j-1, i)
\end{aligned}
$$

$\operatorname{Pr}\left(\right.$ AP $B$ successfully receives a packet $\left.\mid T_{i, j}\right)$

$=i P_{\mathrm{capS}_{A} \rightarrow B}(i-1, j)+j P_{\mathrm{cap}_{B} \rightarrow B}(j-1, i)$

$\operatorname{Pr}\left(\mathrm{A}\right.$ user in User Set A successfully transmits a packet to AP A and AP $B \mid T_{i, j}$ )

$=i P_{\text {aps }_{A} \rightarrow A}(i-1, j) P_{\text {cap }_{A} \rightarrow B}(i-1, j)$

$\operatorname{Pr}\left(\right.$ A user in User Set $B$ succesfully transmits a packet to AP A and AP $B \mid T_{i, j}$ )

$=j P_{\text {cap }_{B} \rightarrow A}(j-1, i) P_{\text {cap }_{B} \rightarrow B}(j-1, i)$

Combining (6)-(9) and (11)-(15) we obtain

$$
\begin{aligned}
\operatorname{Pr}\left(E \mid T_{i, j}\right)=[i( & \left.\left.\frac{1}{R+1}\right)^{i-1}\left(\frac{1}{R \gamma+1}\right)^{j}+j\left(\frac{1}{R+1}\right)^{j-1}\left(\frac{\gamma}{R+\gamma}\right)^{i}\right] \\
\times & {\left[i\left(\frac{1}{R+1}\right)^{i-1}\left(\frac{\gamma}{R+\gamma}\right)^{j}+j\left(\frac{1}{R+1}\right)^{j-1}\left(\frac{1}{R \gamma+1}\right)^{i}\right] } \\
- & {\left[i\left(\frac{1}{R+1}\right)^{i-1}\left(\frac{1}{R \gamma+1}\right)^{j}\left(\frac{1}{R+1}\right)^{i-1}\left(\frac{\gamma}{R+\gamma}\right)^{j}\right.} \\
+ & \left.j\left(\frac{1}{R+1}\right)^{j-1}\left(\frac{\gamma}{R+\gamma}\right)^{i}\left(\frac{1}{R+1}\right)^{j-1}\left(\frac{1}{R \gamma+1}\right)^{i}\right]
\end{aligned}
$$

Considering $\operatorname{Pr}\left(F \mid T_{i, j}\right)$ in (10), we have

$\operatorname{Pr}\left(F \mid T_{i, j}\right)$

$=\operatorname{Pr}\left(\mathrm{A}\right.$ user in User Set A successfully transmits a packet to AP A and AP $\left.B \mid T_{i j}\right)(17)$ $+\operatorname{Pr}\left(\mathrm{A}\right.$ user in User Set B successfully transmits a packet to AP A and AP $B \mid T_{i j}$ )

After combining (6)-(9), (14), (15) and (17), we obtain

$$
\begin{aligned}
\operatorname{Pr}\left(F \mid T_{i, j}\right)= & {\left[i\left(\frac{1}{R+1}\right)^{i-1}\left(\frac{1}{R \gamma+1}\right)^{j}\left(\frac{1}{R+1}\right)^{i-1}\left(\frac{\gamma}{R+\gamma}\right)^{j}\right.} \\
& \left.+j\left(\frac{1}{R+1}\right)^{j-1}\left(\frac{\gamma}{R+\gamma}\right)^{i}\left(\frac{1}{R+1}\right)^{j-1}\left(\frac{1}{R \gamma+1}\right)^{i}\right]
\end{aligned}
$$

We also have

$$
\begin{aligned}
\operatorname{Pr}\left(G \mid T_{i, j}\right)= & \operatorname{Pr}\left(\text { AP A successfully receives a packet } \mid T_{i, j}\right) \\
& \times\left(1-\operatorname{Pr}\left(\text { AP } B \text { successfully receives a packet } \mid T_{i, j}\right)\right)
\end{aligned}
$$

After combining (6)-(9), (12), (13) and (19), we obtain

$$
\begin{aligned}
\operatorname{Pr}\left(G \mid T_{i, j}\right)=[ & \left.i\left(\frac{1}{R+1}\right)^{i-1}\left(\frac{1}{R \gamma+1}\right)^{j}+j\left(\frac{1}{R+1}\right)^{j-1}\left(\frac{\gamma}{R+\gamma}\right)^{i}\right] \\
& \times\left[1-i\left(\frac{1}{R+1}\right)^{i-1}\left(\frac{\gamma}{R+\gamma}\right)^{j}-j\left(\frac{1}{R+1}\right)^{j-1}\left(\frac{1}{R \gamma+1}\right)^{i}\right]
\end{aligned}
$$

Similarly, we are able to obtain

$$
\begin{aligned}
\operatorname{Pr}\left(H \mid T_{i, j}\right)= & {\left[1-i\left(\frac{1}{R+1}\right)^{i-1}\left(\frac{1}{R \gamma+1}\right)^{j}-j\left(\frac{1}{R+1}\right)^{j-1}\left(\frac{\gamma}{R+\gamma}\right)^{i}\right] } \\
& \times\left[i\left(\frac{1}{R+1}\right)^{i-1}\left(\frac{\gamma}{R+\gamma}\right)^{j}+j\left(\frac{1}{R+1}\right)^{j-1}\left(\frac{1}{R \gamma+1}\right)^{i}\right]
\end{aligned}
$$

Finally, the average throughput per access point, $S$, can be obtained by inserting (16), (18), (20) and (21) into (10). 


\section{Delay}

One method to quantify the delay characteristics is to examine the average number of transmission attempts for each successful transmission, which is defined as $A_{\text {avg. }}$. We define $p$ as the probability of a successful reception of a packet when it is transmitted. We have

$$
A_{\mathrm{avg}}=\frac{1}{p}
$$

Let the probability that a user successfully transmits a packet after it is generated is $p_{A}$ or $p_{B}$ when this packet is in User Set $A$ or User Set B. We have

$$
p=p_{A} \frac{N_{A}}{N_{A}+N_{B}}+p_{B} \frac{N_{B}}{N_{A}+N_{B}}
$$

in which

$$
\begin{aligned}
p_{A}= & \sum_{i=0}^{N_{A}-1} \sum_{j=0}^{N_{B}}\left(\begin{array}{c}
N_{A}-1 \\
i
\end{array}\right) \sigma^{i}(1-\sigma)^{N_{A}-1-i}\left(\begin{array}{c}
N_{B} \\
j
\end{array}\right) \sigma^{j}(1-\sigma)^{N_{B}-j} \\
& {\left[\operatorname{Pr}\left(\text { The concerned packet is successfully transmitted to both AP A and AP B|T } T_{i+1, j}\right)\right.} \\
& +\operatorname{Pr}\left(\text { The concerned packet is successfully transmitted to AP A only } \mid T_{i+1, j}\right) \\
& \left.+\operatorname{Pr}\left(\text { The concerned packet is successfully transmitted to AP B only } \mid T_{i+1, j}\right)\right] \\
= & \sum_{i=0}^{N_{A}-1} \sum_{j=0}^{N_{B}}\left(\begin{array}{c}
N_{A}-1 \\
i
\end{array}\right) \sigma^{i}(1-\sigma)^{N_{A}-1-i}\left(\begin{array}{c}
N_{B} \\
j
\end{array}\right) \sigma^{j}(1-\sigma)^{N_{B}-j} \\
& \times\left\{P_{\text {cap } S_{A} \rightarrow A}(i, j) P_{\text {caps } S_{A} \rightarrow B}(i, j)+P_{\text {caps } S_{A} \rightarrow A}(i, j)\left[1-P_{\text {cap } S_{A} \rightarrow B}(i, j)\right]\right. \\
& \left.+P_{\text {cap } S_{A} \rightarrow B}(i, j)\left[1-P_{\text {cap }_{A} \rightarrow A}(i, j)\right]\right\}
\end{aligned}
$$

Inserting (6) and (7) into (24), we obtain

$$
\begin{aligned}
p_{A}=\sum_{i=0}^{N_{A}-1} \sum_{j=0}^{N_{B}}\left(\begin{array}{c}
N_{A}-1 \\
i
\end{array}\right) \sigma^{i}(1-\sigma)^{N_{A}-1-i}\left(\begin{array}{c}
N_{B} \\
j
\end{array}\right) \sigma^{j}(1-\sigma)^{N_{B}-j} \\
+\left\{\left(\frac{1}{R+1}\right)^{i}\left(\frac{1}{R \gamma+1}\right)^{j}\left(\frac{1}{R+1}\right)^{i}\left(\frac{\gamma}{R+\gamma}\right)^{j}\right. \\
+\left(\frac{1}{R+1}\right)^{i}\left(\frac{1}{R \gamma+1}\right)^{j}\left[1-\left(\frac{1}{R+1}\right)^{i}\left(\frac{\gamma}{R+\gamma}\right)^{j}\right] \\
\left.+\left(\frac{1}{R+1}\right)^{i}\left(\frac{\gamma}{R+\gamma}\right)^{j}\left[1-\left(\frac{1}{R+1}\right)^{i}\left(\frac{1}{R \gamma+1}\right)^{j}\right]\right\}
\end{aligned}
$$

Similarly, we are able to find

$$
\begin{aligned}
p_{B}=\sum_{i=0}^{N_{B}-1} & \sum_{j=0}^{N_{A}}\left(\begin{array}{c}
N_{B}-1 \\
i
\end{array}\right) \sigma^{i}(1-\sigma)^{N_{B}-1-i}\left(\begin{array}{c}
N_{A} \\
j
\end{array}\right) \sigma^{j}(1-\sigma)^{N_{A}-j} \\
& \times\left\{\left(\frac{1}{R+1}\right)^{i}\left(\frac{1}{R \gamma+1}\right)^{j}\left(\frac{1}{R+1}\right)^{i}\left(\frac{\gamma}{R+\gamma}\right)^{j}\right. \\
& +\left(\frac{1}{R+1}\right)^{i}\left(\frac{1}{R \gamma+1}\right)^{j}\left[1-\left(\frac{1}{R+1}\right)^{i}\left(\frac{\gamma}{R+\gamma}\right)^{j}\right] \\
& \left.+\left(\frac{1}{R+1}\right)^{i}\left(\frac{\gamma}{R+\gamma}\right)^{j}\left[1-\left(\frac{1}{R+1}\right)^{i}\left(\frac{1}{R \gamma+1}\right)^{j}\right]\right\}
\end{aligned}
$$

Combining (22), (23), (25) and (26), the average number of transmission attempts is obtained.

\section{Special case comparison: no multi-AP diversity}

The following gives the performance results of slotted Aloha without multi-AP diversity in an OM transmit scenario. Following [12] and based on the derivations in Section 3.B, we are able to obtain the throughput as

$$
\begin{aligned}
S= & 0.5 \times\left[\sum_{i=0}^{N_{A}} \sum_{j=0}^{N_{B}}\left(\begin{array}{c}
N_{A} \\
i
\end{array}\right) \sigma^{i}(1-\sigma)^{N_{A}-i}\left(\begin{array}{c}
N_{B} \\
j
\end{array}\right) \sigma^{j}(1-\sigma)^{N_{B}-j_{i}}\left(\frac{1}{R+1}\right)^{i-1}\left(\frac{1}{R \gamma+1}\right)^{j}\right. \\
& \left.+\sum_{i=0}^{N_{B}} \sum_{j=0}^{N_{A}}\left(\begin{array}{c}
N_{B} \\
i
\end{array}\right) \sigma^{i}(1-\sigma)^{N_{B}-i}\left(\begin{array}{c}
N_{A} \\
j
\end{array}\right) \sigma^{j}(1-\sigma)^{N_{A}-j_{i}}\left(\frac{1}{R+1}\right)^{i-1}\left(\frac{1}{R \gamma+1}\right)^{j}\right]
\end{aligned}
$$

The average number of transmission attempts expressed in (22) and (23) still applies with $p_{A}$ and $p_{B}$ as follows,

$$
\begin{aligned}
& p_{A}=\sum_{i=0}^{N_{A}-1} \sum_{j=0}^{N_{B}}\left(\begin{array}{c}
N_{A}-1 \\
i
\end{array}\right) \sigma^{i}(1-\sigma)^{N_{A}-1-i}\left(\begin{array}{c}
N_{B} \\
j
\end{array}\right) \sigma^{j}(1-\sigma)^{N_{B}-j}\left(\frac{1}{R+1}\right)^{i}\left(\frac{1}{R \gamma+1}\right)^{j} \\
& p_{B}=\sum_{i=0}^{N_{B}-1} \sum_{j=0}^{N_{A}}\left(\begin{array}{c}
N_{B}-1 \\
i
\end{array}\right) \sigma^{i}(1-\sigma)^{N_{B}-1-i}\left(\begin{array}{c}
N_{A} \\
j
\end{array}\right) \sigma^{j}(1-\sigma)^{N_{A}-j}\left(\frac{1}{R+1}\right)^{i}\left(\frac{1}{R \gamma+1}\right)^{j}
\end{aligned}
$$

\section{Slotted Aloha with Multi-AP Diversity and BF \\ Antenna}

\section{A. Capture probability}

In order to investigate the capture effect in this multiAP diversity and BF scenario, we define a function

$$
f(I, J, \Gamma)=\operatorname{Pr}\left(\frac{x}{\sum_{i=1}^{I} y_{i}+\sum_{j=1}^{J} z_{j}}>R, x>\tilde{x}, y_{i}>\tilde{y}_{i}, z_{j}>\tilde{z}_{j}\right)
$$

where $x, y_{i}$, and $z_{j}$ are the received power of the desired packet, the received power of interfering packets from the same user set as the desired packet, and the received power of interfering packets from the different user set as the desired packet, and respectively, for a target AP; $\tilde{x}$ is the received power of the desired packet if the desired packet is received at the AP other than the target AP. $\tilde{y}_{i}$ and $\tilde{z}_{j}$ are similarly defined. We let

$$
\frac{E[\tilde{x}]}{E[x]}=\frac{E\left[\tilde{y}_{i}\right]}{E\left[y_{i}\right]}=\frac{E\left[z_{j}\right]}{E\left[\tilde{z}_{j}\right]}=\Gamma
$$

For examples, $f(m-1, n, \gamma)$ denotes the probability that for a given AP (say AP A), $m$ transmitting users of user set $\mathrm{A}$ and $n$ transmitting users of user set $\mathrm{B}$ choose AP A and one of the $m$ users successfully captures AP A; $f\left(m-1, n, \frac{1}{\gamma}\right)$ denotes the probability that for a given AP (say AP A), $m$ transmitting users of user set $\mathrm{B}$ and $n$ transmitting users of user set A choose AP A and one of the $m$ users successfully captures AP A. The following equation derives this function.

$$
\begin{aligned}
& f(I, J, \Gamma) \\
& =\prod_{i=1}^{I} \int_{\tilde{y}_{i}}^{\infty} \frac{1}{\mu} e^{-\frac{y_{i}}{\mu}} d y_{i} \prod_{j=1}^{I} \int_{\tilde{z}_{j}}^{\infty} \frac{1}{v} e^{-\frac{z_{j}}{v}} d z_{j} \prod_{i=1}^{I} \int_{0}^{\infty} \frac{1}{v} e^{-\frac{\tilde{y}_{i}}{v}} d \tilde{y}_{i} \prod_{j=1}^{I} \int_{0}^{\infty} \frac{1}{Z_{2}} e^{-\frac{\tilde{z}_{j}}{\mu}} d \tilde{z}_{j} \\
& \times\left[\int_{R\left(\sum_{i=1}^{d} y_{i}+\sum_{j=1}^{\prime} z_{j}\right)} \frac{1}{\mu} e^{-\frac{x}{\mu}} d x \int_{0}^{R\left(\sum_{i=1}^{l} y_{i}+\sum_{j=1}^{\prime} z_{j}\right)} \frac{1}{v} e^{-\frac{\tilde{x}}{v}} d \tilde{x}+\int_{\tilde{x}}^{\infty} \frac{1}{\mu} e^{-\frac{x}{\mu}} d x\right. \\
& \left.\int_{R\left(\sum_{i=1}^{l} x_{i}+\sum_{j=1}^{\prime} y_{j}\right)}^{\infty} \frac{1}{v} e^{-\frac{\tilde{x}}{v}} d \tilde{x}\right] \\
& =\frac{1}{[(1+R)(1+\Gamma+R \Gamma)]^{1}} \frac{1}{\left[(1+R \Gamma)\left(1+\frac{1}{\Gamma}+R\right)\right]^{\prime}}-\frac{1}{\frac{1}{\Gamma}+1} \\
& \times \frac{1}{\left\{\left[1+R\left(1+\frac{1}{\Gamma}\right)[[1+\Gamma+R(\Gamma+1)]\}^{I}\right.\right.} \frac{1}{\left\{[1+R(\Gamma+1)]\left[1+\frac{1}{\Gamma}+R\left(1+\frac{1}{\Gamma}\right)\right]\right\}^{\prime}}
\end{aligned}
$$




\section{B. Throughput}

To calculate the average throughput per access point in the BF cases, we can still use the modeling approach based on the event $T_{i j}$ as defined in Section 3.B. Furthermore, a new event $Q_{m, n}$ is defined below.

$Q_{m, n}: m$ transmitting users in User Set $A$ choose AP $A$ and $n$ transmitting users in User Set $B$ choose AP $A$ as their server.

The throughput of AP $A, S_{a}$, can be calculated as follows.

$$
\begin{aligned}
S_{a}= & \sum_{i=0}^{N_{A}} \sum_{j=0}^{N_{B}}\left(\begin{array}{c}
N_{A} \\
i
\end{array}\right) \sigma^{i}(1-\sigma)^{N_{A}-i}\left(\begin{array}{c}
N_{B} \\
j
\end{array}\right) \sigma^{j}(1-\sigma)^{N_{B}-j} \operatorname{Pr}(\text { AP A successfully } \\
& \text { receives a packet } \left.\mid T_{i, j}\right) \\
= & \sum_{i=0}^{N_{A}} \sum_{j=0}^{N_{B}}\left(\begin{array}{c}
N_{A} \\
i
\end{array}\right) \sigma^{i}(1-\sigma)^{N_{A}-i}\left(\begin{array}{c}
N_{B} \\
j
\end{array}\right) \sigma^{j}(1-\sigma)^{N_{B}-j} \sum_{m=0}^{i} \sum_{n=0}^{j} \operatorname{Pr}\left(Q_{m, n} \mid T_{i, j}\right) \\
& \left.\times \operatorname{Pr} \text { AP A successfully receives a packet } \mid T_{i, j} Q_{m, n}\right)
\end{aligned}
$$

Expanding the conditional probability $\operatorname{Pr}\left(Q_{m, n} \mid T_{i, j}\right)$, the throughput of AP A is expressed as

$$
\begin{aligned}
S_{a}= & \sum_{i=0}^{N_{A}} \sum_{j=0}^{N_{B}}\left(\begin{array}{c}
N_{A} \\
i
\end{array}\right) \sigma^{i}(1-\sigma)^{N_{A}-i}\left(\begin{array}{c}
N_{B} \\
j
\end{array}\right) \sigma^{j}(1-\sigma)^{N_{B}-j} \sum_{m=0}^{i} \sum_{n=0}^{j}\left(\begin{array}{c}
i \\
m
\end{array}\right)\left(\begin{array}{l}
j \\
n
\end{array}\right) \\
& \times(\operatorname{Pr}(\text { a transmitting user in User Set } A \text { chooses AP A }))^{m} \\
& \times\left(\operatorname{Pr}(\text { a transmitting user in User Set B chooses AP A) })^{n}\right. \\
& \times(1-\operatorname{Pr} \text { a transmitting user in User Set A chooses AP A }))^{i-m} \\
& \times(1-\operatorname{Pr} \text { a transmitting user in User Set } B \text { chooses AP A }))^{j-n} \\
& \times \operatorname{Pr}\left(\text { AP A successfully receives one packet } \mid T_{i, j} Q_{m, n}\right)
\end{aligned}
$$

Notice

$\operatorname{Pr}\left(\mathrm{AP} A\right.$ successfully receives one packet $\mid T_{i, j} \mathrm{Q}_{m, n}$ )

$=\operatorname{Pr}\left(\mathrm{AP} A\right.$ successfully receives one packet from User Set $\left.A \mid T_{i, j} Q_{m, n}\right)$

$+\operatorname{Pr}\left(\mathrm{AP} A\right.$ successfully receives one packet from User Set $\left.B \mid T_{i, j} \mathrm{Q}_{m, n}\right)$

and

$(\operatorname{Pr}(\text { a transmitting users in User Set A chooses AP A }))^{m}$

$\times(\operatorname{Pr}(\text { a transmitting users in User Set } B \text { chooses AP } A))^{n}$

$\times \operatorname{Pr}\left(\mathrm{AP} A\right.$ successfully receives one packet from User Set $\left.A \mid T_{i, j} Q_{m, n}\right)$

$=m \operatorname{Pr}\left(\frac{x}{\sum_{i=1}^{m-1} \gamma_{i}+\sum_{j=1}^{n} z_{j}}>R \mid x>\tilde{x}_{,} y_{i}>\tilde{y}_{i}, z_{j}>\tilde{z}_{j}\right) \operatorname{Pr}\left(x>\tilde{x}_{,} y_{i}>\tilde{y}_{i}, z_{j}>\tilde{z}_{j}\right)$

$=m f(m-1, n, \gamma)$

Similarly, we are able to obtain

$(\operatorname{Pr}(\text { a transmitting users in User Set A chooses AP A }))^{m}$

$\times(\operatorname{Pr}(\text { a transmitting users in User Set } B \text { chooses AP } A))^{n}$

$\times \operatorname{Pr}\left(\right.$ AP A successfully receives one packet from User Set $\left.B \mid T_{i, j} Q_{m, n}\right)(37)$

$=n f\left(n-1, m, \frac{1}{\gamma}\right)$

Inserting (34)-(36) into (33), we obtain

$$
\begin{aligned}
S_{a}= & \sum_{i=0}^{N_{A}} \sum_{j=0}^{N_{B}}\left(\begin{array}{c}
N_{A} \\
i
\end{array}\right) \sigma^{i}(1-\sigma)^{N_{A}-i}\left(\begin{array}{c}
N_{B} \\
j
\end{array}\right) \sigma^{j}(1-\sigma)^{N_{B}-j} \\
& \times \sum_{m=0}^{i} \sum_{n=0}^{j}\left(\begin{array}{c}
i \\
m
\end{array}\right)\left(\begin{array}{c}
j \\
n
\end{array}\right)\left(m f(m-1, n, \gamma)+n f\left(n-1, m, \frac{1}{\gamma}\right)\right. \\
& \times(1-\operatorname{Pr}(\text { a transmitting users in User Set A chooses AP A }))^{i-m} \\
& \times(1-\operatorname{Pr}(\text { a transmitting users in User Set } B \text { chooses AP A }))^{j-n}
\end{aligned}
$$

Following the derivations in (5), we get

$$
\operatorname{Pr}(\text { a transmitting user in User Set } A \text { chooses AP A })=\frac{X}{\mathcal{X}+X}
$$

and

$$
\operatorname{Pr}(\text { a transmitting user in User Set } B \text { chooses } \mathrm{AP} A)=\frac{\mathcal{X}}{X+\mathcal{X}}
$$

Using (2)-(4) and inserting (31), (38), (39) into (37), we obtain

$$
\begin{aligned}
S_{a}= & \sum_{i=0}^{N_{A}} \sum_{j=0}^{N_{B}}\left(\begin{array}{c}
N_{A} \\
i
\end{array}\right) \sigma^{i}(1-\sigma)^{N_{A}-i}\left(\begin{array}{c}
N_{B} \\
j
\end{array}\right) \sigma^{j}(1-\sigma)^{N_{B}-j} \\
& \times \sum_{m=0}^{i} \sum_{n=0}^{j}\left(\begin{array}{c}
i \\
m
\end{array}\right)\left(\begin{array}{c}
j \\
n
\end{array}\right)\left\{\frac{m}{[(1+R)(1+\gamma+R \gamma)]^{m-1}\left[(1+R \gamma)\left(1+\frac{1}{\gamma}+R\right)\right]^{n}}\right. \\
& -\frac{m \gamma}{(1+\gamma)\left[(R+1)\left(1+R\left(1+\frac{1}{\gamma}\right)\right)(1+\gamma)\right]^{m-1}\left[(R+1)(1+R(1+\gamma))\left(1+\frac{1}{\gamma}\right)\right]^{n}} \\
& +\frac{n}{\left[(1+R)\left(1+\frac{1}{\gamma}+\frac{R}{\gamma}\right)\right]^{n-1}\left[\left(1+\frac{R}{\gamma}\right)(1+\gamma+R)\right]^{m}} \\
& \left.-\frac{n}{(1+\gamma)\left[(R+1)(1+R(1+\gamma))\left(1+\frac{1}{\gamma}\right)\right]^{n-1}\left[(R+1)\left(1+R\left(1+\frac{1}{\gamma}\right)\right)(1+\gamma)\right]^{m}}\right\} \\
& \times\left(\frac{\gamma}{\gamma+1}\right)^{i-m}\left(\frac{1}{1+\gamma}\right)^{j-n}
\end{aligned}
$$

Following a similar derivation process as (32)-(40), we obtain the throughput of access point $B, S_{b}$, as

$$
\begin{aligned}
S_{b}= & \sum_{i=0}^{N_{B}} \sum_{j=0}^{N_{A}}\left(\begin{array}{c}
N_{B} \\
i
\end{array}\right) \sigma^{i}(1-\sigma)^{N_{B}-i}\left(\begin{array}{c}
N_{A} \\
j
\end{array}\right) \sigma^{j}(1-\sigma)^{N_{A}-j} \\
& \times \sum_{m=0}^{i} \sum_{n=0}^{j}\left(\begin{array}{c}
i \\
m
\end{array}\right)\left(\begin{array}{c}
j \\
n
\end{array}\right)\left\{\frac{m}{[(1+R)(1+\gamma+R \gamma)]^{m-1}\left[(1+R \gamma)\left(1+\frac{1}{\gamma}+R\right)\right]^{n}}\right. \\
& -\frac{m \gamma}{(1+\gamma)\left[(R+1)\left(1+R\left(1+\frac{1}{\gamma}\right)\right)(1+\gamma)\right]^{m-1}\left[(R+1)(1+R(1+\gamma))\left(1+\frac{1}{\gamma}\right)\right]^{n}} \\
& +\frac{n}{\left[(1+R)\left(1+\frac{1}{\gamma}+\frac{R}{\gamma}\right)\right]^{n-1}\left[\left(1+\frac{R}{\gamma}\right)(1+\gamma+R)\right]^{m}} \\
& \left.-\frac{n}{(1+\gamma)\left[(R+1)(1+R(1+\gamma))\left(1+\frac{1}{\gamma}\right)\right]^{n-1}\left[(R+1)\left(1+R\left(1+\frac{1}{\gamma}\right)\right)(1+\gamma)\right]^{m}}\right\} \\
& \times\left(\frac{\gamma}{\gamma+1}\right)^{i-m}\left(\frac{1}{1+\gamma}\right)^{j-n}
\end{aligned}
$$

The average throughput per AP, $S$, is thus $\frac{S_{a}+S_{b}}{2}$.

\section{Delay}

The derivation of the delay in the BF case is similar to that in the OM case. We use the parameters $p, p_{A}$, $p_{B}$ defined in Section 3.C and event $T_{i, j}$ defined in Section 3.B. The user transmitting a concerned packet is referred to as a concerned user and all other users are called non-concerned users. Furthermore, a new event $J_{m, n}$ is defined below.

$J_{m, n}$ : Excluding the concerned user, $m$ transmitting users in User Set $A$ choose AP $A$ and $n$ transmitting users in User Set $B$ choose AP $A$ as their server.

We have

$$
\begin{aligned}
p_{A}= & \sum_{i=0}^{N_{A}-1} \sum_{j=0}^{N_{B}}\left(\begin{array}{c}
N_{A}-1 \\
i
\end{array}\right) \sigma^{i}(1-\sigma)^{N_{A}-1-i}\left(\begin{array}{c}
N_{B} \\
j
\end{array}\right) \sigma^{j}(1-\sigma)^{N_{B}-j} \\
& \times \operatorname{Pr}\left(\text { P A or AP B successfully receives the concerned packet } \mid T_{i+1, j}\right) \\
= & \sum_{i=0}^{N_{A}-1} \sum_{j=0}^{N_{B}}\left(\begin{array}{c}
N_{A}-1 \\
i
\end{array}\right) \sigma^{i}(1-\sigma)^{N_{A}-1-i}\left(\begin{array}{c}
N_{B} \\
j
\end{array}\right) \sigma^{j}(1-\sigma)^{N_{B}-j} \sum_{m=0}^{i} \sum_{n=0}^{j} \operatorname{Pr}\left(J_{m, n} \mid T_{i+1, j}\right) \\
& \times \operatorname{Pr}\left(\text { AP A or AP B successfully receives the concerned packet } \mid T_{i+1, j} j_{m, n}\right)
\end{aligned}
$$


Expanding $\operatorname{Pr}\left(J_{m, n} \mid T_{i+1, j}\right)$ and $\operatorname{Pr}(\mathrm{AP} A$ or AP $B$ successfully receives the concerned packet $\left.\mid T_{i+1, j} J_{m, n}\right)$, we have

$$
\begin{aligned}
p_{A}= & \sum_{i=0}^{N_{A}-1} \sum_{j=0}^{N_{B}}\left(\begin{array}{c}
N_{A} \\
i
\end{array}\right) \sigma^{i}(1-\sigma)^{N_{A}-i-1}\left(\begin{array}{c}
N_{B} \\
j
\end{array}\right) \sigma^{j}(1-\sigma)^{N_{B}-j} \sum_{m=0}^{i} \sum_{n=0}^{j}\left(\begin{array}{c}
i \\
m
\end{array}\right)\left(\begin{array}{c}
j \\
n
\end{array}\right) \\
& \times(\operatorname{Pr} \text { (a non - concerned transmitting user in User Set A chooses AP A }))^{m} \\
& \times\left(\operatorname{Pr}(\text { a non - concerned transmitting user in User Set B chooses AP A) })^{n}\right. \\
& \times(1-\operatorname{Pr} \text { a non - concerned transmitting user in User Set A chooses AP A) })^{i-m} \\
& \times(1-\operatorname{Pr}(\text { a non - concerned transmitting user in } \text { User Set } B \text { chooses AP } A))^{j-n} \\
& \times\left[\operatorname{Pr}\left(\text { AP A successfully receives the concerned packet } \mid T_{i+1, j} J_{m, n}\right)\right. \\
& \left.+\operatorname{Pr}\left(\mathrm{AP} B \text { successfully receives the concerned packet } \mid T_{i+1, j} J_{m, n}\right)\right]
\end{aligned}
$$

Notice that

(Pr(a non - concerned transmitting user in User Set A chooses AP A $))^{m}$

$\times(\operatorname{Pr}(\text { a non - concerned transmitting user in User Set } B \text { chooses AP } A))^{n}$

$\times \operatorname{Pr}\left(\mathrm{AP} A\right.$ successfully receives the concerned packet $\left.\left.\mid T_{i+1, j} J_{m, n}\right)\right)$

$$
\begin{aligned}
& =\operatorname{Pr}\left(\frac{x}{\sum_{i=1}^{m} y_{i}+\sum_{j=1}^{n} z_{j}}>R, x>\tilde{x} \mid y_{i}>\tilde{y}_{i}, z_{j}>\tilde{z}_{j}\right) \operatorname{Pr}\left(y_{i}>\tilde{y}_{i}, z_{j}>\tilde{z}_{j}\right) \\
& =f(m, n, \gamma)
\end{aligned}
$$

Similarly, we have

$$
\begin{aligned}
& (1-\operatorname{Pr}(\text { a non - concerned transmitting user in User Set A chooses AP A }))^{i-m} \\
& \times(1-\operatorname{Pr}(\text { a non }- \text { concerned transmitting user in User Set } B \text { chooses AP A }))^{j-n} \\
& \left.+\operatorname{Pr}\left(\mathrm{AP} B \text { successfully receives the concerned packet } \mid T_{i+1, j} J_{m, n}\right)\right) \\
= & f\left(i-m, j-n, \frac{1}{\gamma}\right)
\end{aligned}
$$

Inserting (38), (39), (44), (45) into (43) and using (2)(4) and the function defined in (31), (43) can be rewritten as

$$
\begin{aligned}
p_{A}= & \sum_{i=0}^{N_{A}-1} \sum_{j=0}^{N_{B}}\left(\begin{array}{c}
N_{A}-1 \\
i
\end{array}\right) \sigma^{i}(1-\sigma)^{N_{A}-1-i}\left(\begin{array}{c}
N_{B} \\
j
\end{array}\right) \sigma^{j}(1-\sigma)^{N_{B}-j} \sum_{m=0}^{i} \sum_{n=0}^{j}\left(\begin{array}{c}
i \\
m
\end{array}\right)\left(\begin{array}{l}
j \\
n
\end{array}\right) \\
& \times\left\{( \frac { \gamma } { \gamma + 1 } ) ^ { i - m } ( \frac { 1 } { 1 + \gamma } ) ^ { j - n } \left\{\frac{1}{[(1+R)(1+\gamma+R \gamma)]^{m}\left[(1+R \gamma)\left(1+\frac{1}{\gamma}+R\right)\right]^{n}}\right.\right. \\
& \left.-\frac{\gamma}{(1+\gamma)\left[(R+1)\left(1+R\left(1+\frac{1}{\gamma}\right)\right)(1+\gamma)\right]^{m}\left[(R+1)(1+R(1+\gamma))\left(1+\frac{1}{\gamma}\right)\right]^{n}}\right\} \\
& +\left(\frac{1}{\gamma+1}\right)^{m}\left(\frac{\gamma}{1+\gamma}\right)^{n}\left\{\frac{1}{\left[(1+R)\left(1+\frac{1}{\gamma}+\frac{R}{\gamma}\right)\right]^{i-m}\left[\left(1+\frac{R}{\gamma}\right)(1+\gamma+R)\right]^{j-n}}\right. \\
& \left.\left.-\frac{1}{(1+\gamma)\left[(R+1)(1+R(1+\gamma))\left(1+\frac{1}{\gamma}\right)\right]^{i-m}\left[(R+1)\left(1+R\left(1+\frac{1}{\gamma}\right)\right)(1+\gamma)\right]^{j-n}}\right\}\right\}
\end{aligned}
$$

The probability $p_{B}$ can be similarly found as

$$
\begin{aligned}
p_{B}= & \sum_{i=0}^{N_{B}-1} \sum_{j=0}^{N_{A}}\left(\begin{array}{c}
N_{B}-1 \\
i
\end{array}\right) \sigma^{i}(1-\sigma)^{N_{B}-1-i}\left(\begin{array}{c}
N_{A} \\
j
\end{array}\right) \sigma^{j}(1-\sigma)^{N_{A}-j} \sum_{m=0}^{i} \sum_{n=0}^{j}\left(\begin{array}{c}
i \\
m
\end{array}\right)\left(\begin{array}{c}
j \\
n
\end{array}\right) \\
& \times\left\{( \frac { \gamma } { \gamma + 1 } ) ^ { i - m } ( \frac { 1 } { 1 + \gamma } ) ^ { j - n } \left\{\frac{1}{[(1+R)(1+\gamma+R \gamma)]^{m}\left[(1+R \gamma)\left(1+\frac{1}{\gamma}+R\right)\right]^{n}}\right.\right. \\
& \left.-\frac{\gamma}{(1+\gamma)\left[(R+1)\left(1+R\left(1+\frac{1}{\gamma}\right)\right)(1+\gamma)\right]^{m}\left[(R+1)(1+R(1+\gamma))\left(1+\frac{1}{\gamma}\right)\right]^{n}}\right\} \\
& +\left(\frac{1}{\gamma+1}\right)^{m}\left(\frac{\gamma}{1+\gamma}\right)^{n}\left\{\frac{1}{\left[(1+R)\left(1+\frac{1}{\gamma}+\frac{R}{\gamma}\right)\right]^{i-m}\left[\left(1+\frac{R}{\gamma}\right)(1+\gamma+R)\right]^{j-n}}\right. \\
& \left.\left.-\frac{1}{(1+\gamma)\left[(R+1)(1+R(1+\gamma))\left(1+\frac{1}{\gamma}\right)\right]^{i-m}\left[(R+1)\left(1+R\left(1+\frac{1}{\gamma}\right)\right)(1+\gamma)\right]^{j-n}}\right\}\right\}
\end{aligned}
$$

Applying $p_{A}$ and $p_{B}$ into (22) and (23), the average number of transmission attempts is obtained.

\section{Special case comparison: no Multi-AP diversity}

The following presents the throughput and delay expressions considering BF but without multi-AP diversity. Following [19], we are able to obtain the throughput as

$$
S=0.5 \times\left[\sum_{i=0}^{N_{A}}\left(\begin{array}{c}
N_{A} \\
i
\end{array}\right) \sigma^{i}(1-\sigma)^{N_{A}-i} \frac{i}{(R+1)^{i-1}}+\sum_{j=0}^{N_{B}}\left(\begin{array}{c}
N_{B} \\
j
\end{array}\right) \sigma^{j}(1-\sigma)^{N_{B}-j} \frac{j}{(R+1)^{j-1}}\right]
$$

The delay expression follows (22) and (23), with the probabilities $p_{A}$ and $p_{B}$ given as

$$
\begin{aligned}
& p_{A}=\sum_{i=0}^{N_{A}-1}\left(\begin{array}{c}
N_{A}-1 \\
i
\end{array}\right) \sigma^{i}(1-\sigma)^{N_{A}-1-i} \frac{1}{(R+1)^{i}} \\
& p_{B}=\sum_{j=0}^{N_{B}}\left(\begin{array}{c}
N_{B}-1 \\
i
\end{array}\right) \sigma^{j}(1-\sigma)^{N_{B}-1-j} \frac{1}{(R+1)^{j}}
\end{aligned}
$$

\section{Numerical Results: Theoretical and Simulation}

Numerical results presented in this section are mostly based on theoretical formulas. For the comparison purpose, a number of simulation results are also presented. All simulation results are obtained by running MATLAB programs for 500000 time slots. Rayleigh fading and independent transmission links are assumed in generating signal strength values. For packet arrivals, a Poisson distribution is used in determining the number of packets generated in each time slot. Signaling is not implemented in the simulation, assuming that all acknowledgments are received successfully.

Figure 3 compares the throughput of slotted Aloha when BF with multi-AP diversity and OM with multiAP diversity are used. Both analytical and simulation results are presented. System parameters considered include $N_{A}=N_{B}=25, \gamma=0.1$, and $R=3 \mathrm{~dB}$. Numerical results illustrate that the analytical evaluation and simulation results match very well. The scenario with BF clearly outperforms the OM case under high traffic load

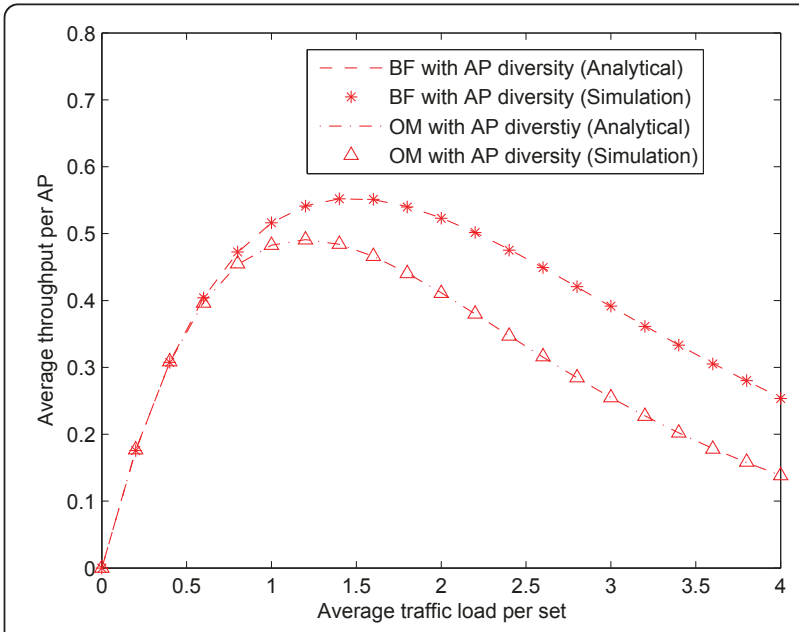

Figure 3 Throughput comparison: $O M$ versus $B F$, with AP diversity; analytical versus simulation results, $N_{A}=N_{B}=25, \gamma=$ $0.1, R=3 \mathrm{~dB}$. 
conditions with an approximately $12 \%$ improvement in peak throughput.

Figure 4 considers the $\mathrm{OM}$ case and examines the impact of the capture ratio, $R$. System parameters $N_{A}$ and $N_{B}$ are assumed to be 25 and $\gamma$ is assumed to be 0.1 . It is seen that a lower capture ratio leads to higher throughput. The OM case with AP diversity consistently outperforms that without AP diversity, especially when the capture ratio is small.

Figure 5 considers the $\mathrm{OM}$ case and examines the impact of $\gamma$ values (see (4)). System parameters $N_{A}$ and $N_{B}$ are assumed to be 25 and $R$ is assumed to be $3 \mathrm{~dB}$. The throughput decreases as $\gamma$ increases (due to more interference between the two APs). It is also noted that the throughput gain due to multi-AP diversity is more significant when $\gamma$ is larger.

Figure 6 examines the impact of user distributions $\left(N_{A}\right.$ versus $\left.N_{B}\right)$ in the OM case with multi-AP diversity. The system parameter $\gamma$ is assumed to be 0.1 and $R$ is assumed to be $3 \mathrm{~dB}$. The scenario with even user distributions $\left(N_{A}=25\right.$ and $\left.N_{B}=25\right)$ outperforms other scenarios with uneven distributions. When the user distributions become very uneven (e.g., $N_{A}=40$ and $N_{B}=$ $10)$, throughput is noticeably lower due to the potential of a higher collision probability at the heavy-load AP $\left(N_{A}=40\right)$.

Figure $7 \mathrm{a}, \mathrm{b}, \mathrm{c}$ considers the BF scenario and examines the impact of multi-AP diversity. System parameter $\gamma$ is assumed to be 0.1 and $R$ is assumed to be $3 \mathrm{~dB}$. The figures show that the advantage, if any, of multi-AP diversity in the BF case depends on the user distributions between the two user sets. When the distributions are extremely uneven (e.g., $N_{A}=45$ and $N_{B}=5$ ), the multi-

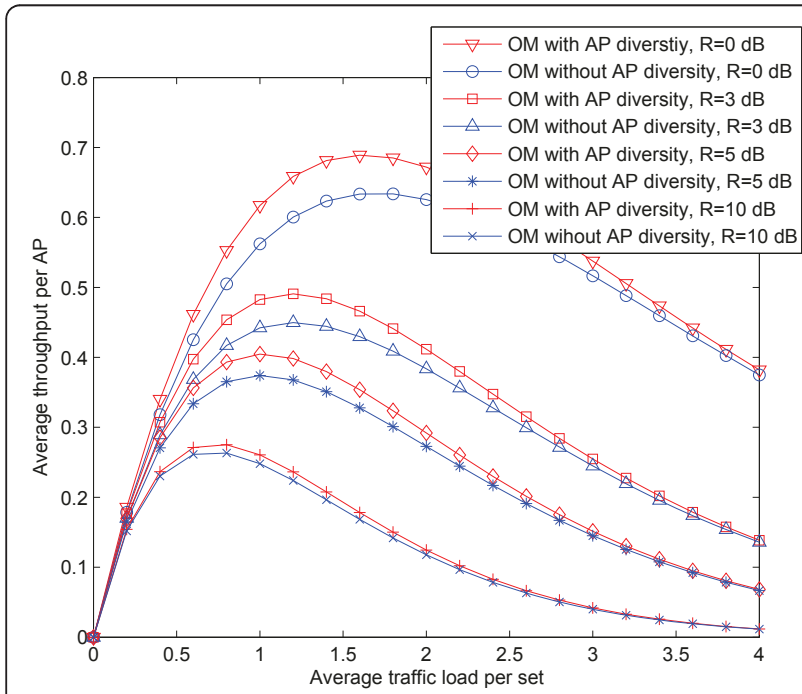

Figure 4 Throughput of OM with different $R$ values, $N_{A}=N_{B}=$ $25, \gamma=0.1$.

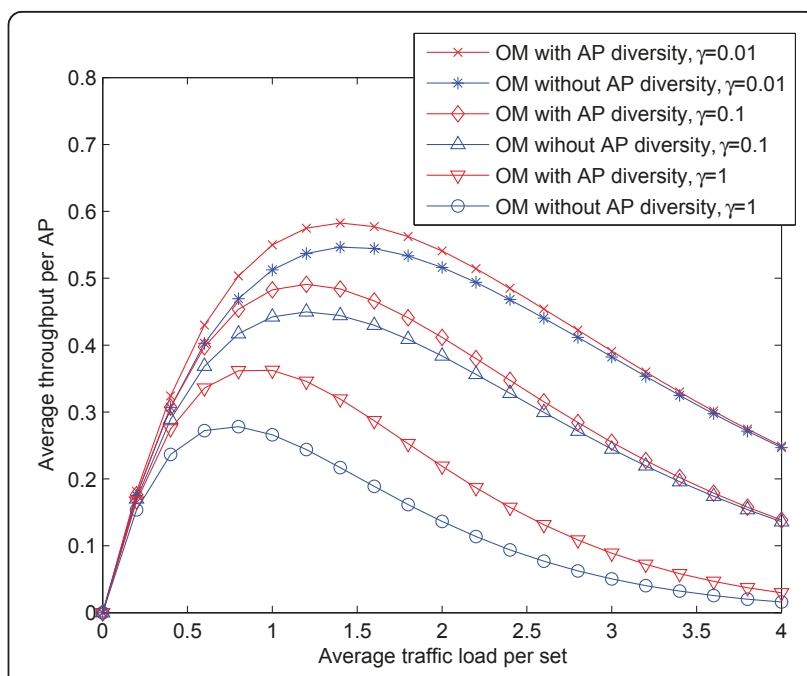

Figure 5 Throughput of $O M$ with different $\gamma$ values, $N_{A}=N_{B}=$ $25, R=3 \mathrm{~dB}$.

AP diversity clearly shows its advantage. When the distributions become less uneven (e.g., $N_{A}=40$ and $N_{B}=$ 10), the advantage of multi-AP diversity is seen for a wide traffic load range, but not for extremely high traffic load conditions. When the user distributions become even (e.g., $N_{A}=25$ and $N_{B}=25$ ), the advantage of multiAP diversity disappears. These observations are due to a traffic redistribution characteristics of AP diversity. When the user distribution is uneven, with AP diversity, some users could effectively migrate from the AP with a heavy load to the AP with a light load, which may lead to an overall performance improvement. However, when the user distribution is even, AP diversity may cause a situation where one AP gets overly loaded, which brings down overall throughput.

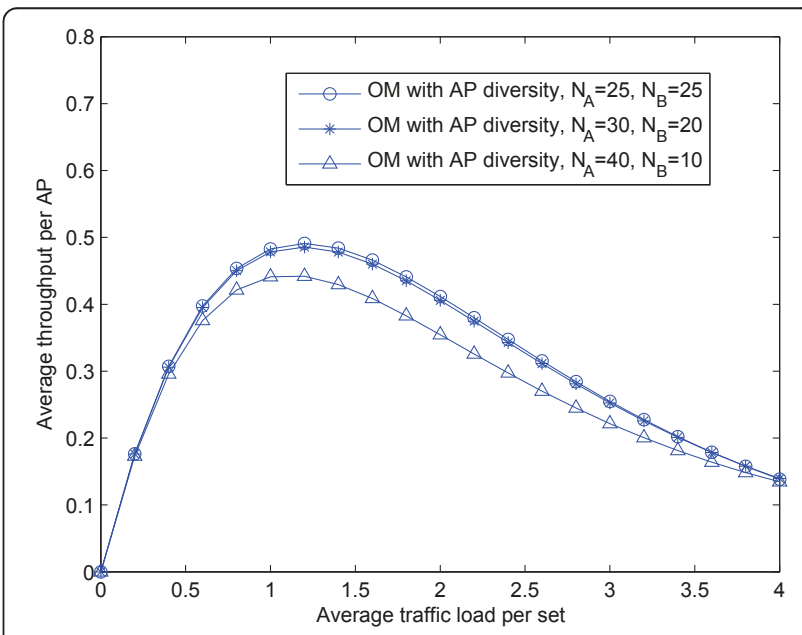

Figure 6 Throughput of OM with different user distributions, $\gamma$ $=0.1, R=3 \mathrm{~dB}$. 


$$
\left[N_{A}=45, N_{B}=5 .\right]
$$
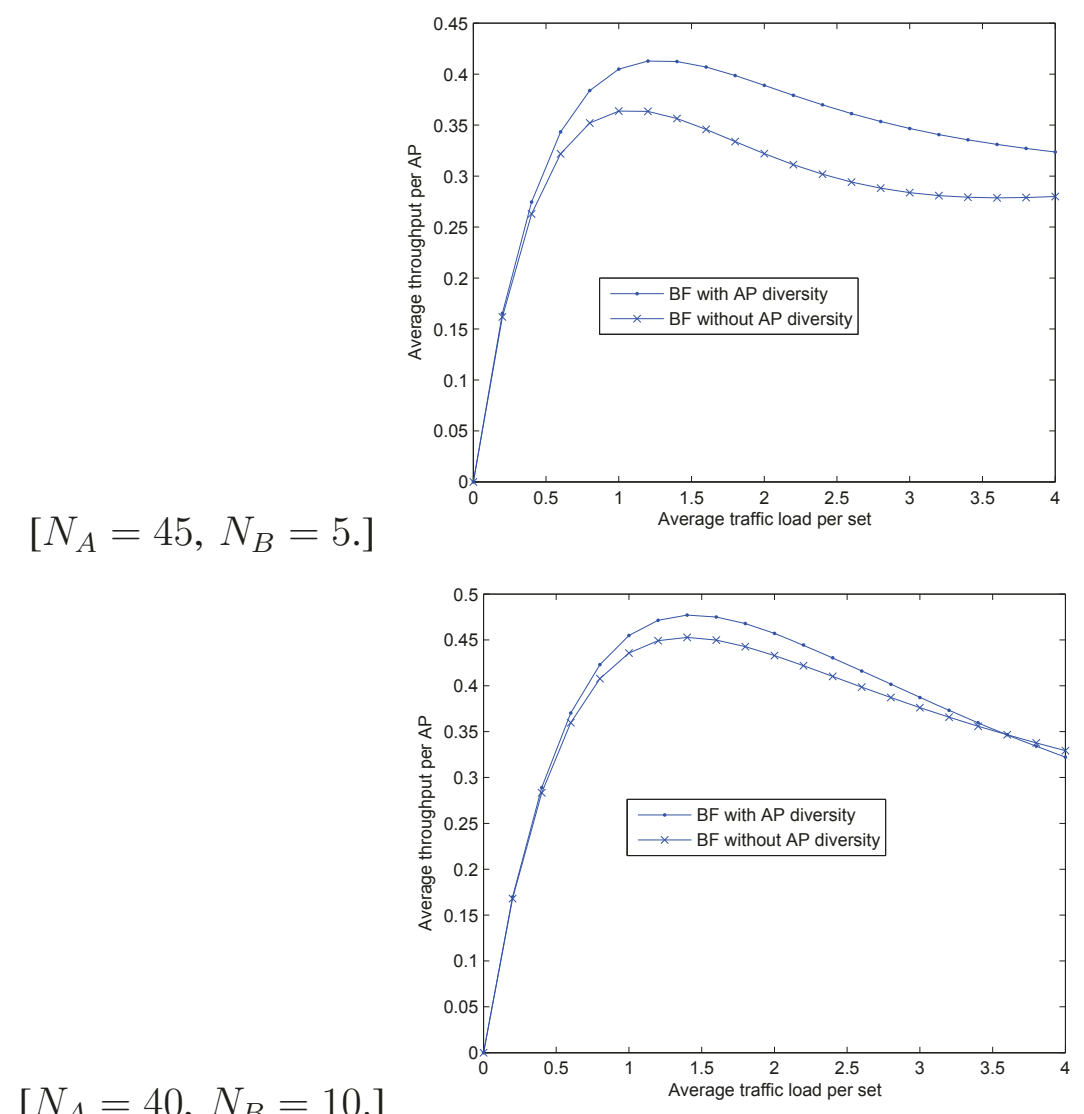

$\left[N_{A}=40, N_{B}=10.\right]$

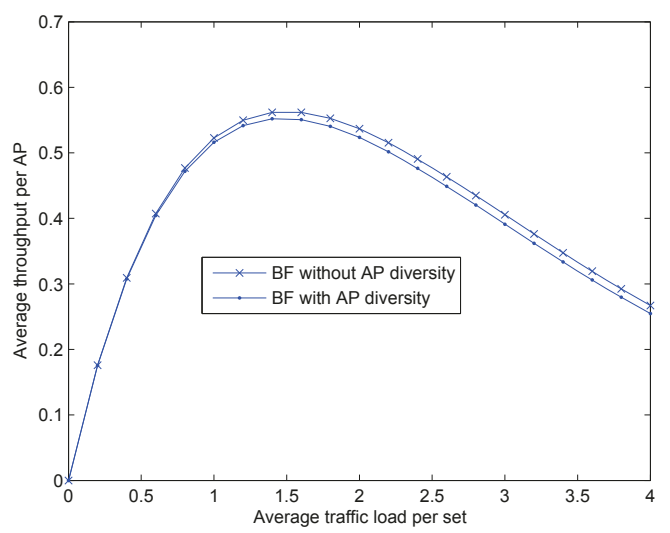

$\left[N_{A}=25, N_{B}=25.\right]$

Figure 7 Throughput of BF with different user distributions, $\gamma=0.1$ and $R=3 \mathrm{~dB}$

One method to study the delay performance is to examine the average number of transmission attempts for each successful packet transmission. In Figure 8, $\mathrm{OM}$ with multi-AP diversity and BF with multi-AP diversity are compared in terms of the average number of transmission attempts for each successful transmission. System parameters considered include $N_{A}=25$, $N_{B}=25, \gamma=0.1$, and $R=3 \mathrm{~dB}$. Both analytical and simulation results are presented in Figure 8 and the analytical evaluation and simulation match very well. Figure
8 , which illustrates that BF with multi-AP diversity outperforms OM with multi-AP diversity in the delay performance.

\section{Conclusions}

This paper investigates the impact of multi-AP diversity and $\mathrm{BF}$ in slotted Aloha. A total of four network scenarios are examined, i.e., OM with multi-AP diversity, OM without multi-AP diversity, BF with multi-AP diversity and BF without multi-AP diversity. Performance 


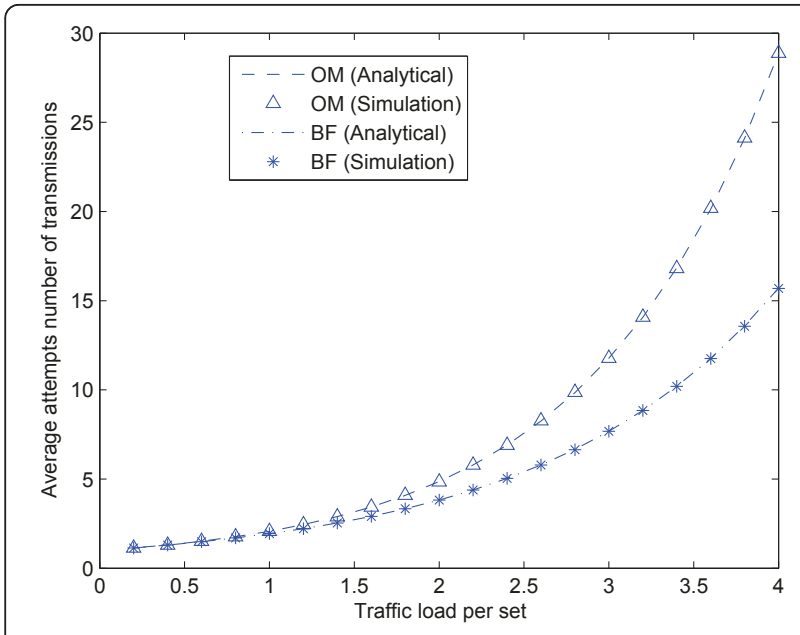

Figure 8 Average number of transmission attempts for a successful packet transmission: $O M$ versus $B F$, with $A P$ diversity; analytical versus simulation results, $N_{A}=N_{B}=25, \gamma=0.1, R=3 \mathrm{~dB}$.

evaluations conclude that, for OM systems, a configuration with multi-AP diversity always outperforms that without multi-AP diversity (Figures 4 and 5 ). For BF systems, multi-AP diversity provides performance advantages only under conditions with extremely uneven user distributions (Figure 7). Considering multi-AP diversity, BF systems outperform OM systems in terms of throughput and delay (Figures 3 and 8).

\section{Competing interests}

The authors declare that they have no competing interests.

\section{Abbreviations}

AP: access points; BF: beamforming; multi-AP: multi-access-point; OM: omnidirectional.

Received: 16 November 2010 Accepted: 5 October 2011

Published: 5 October 2011

\section{References}

1. N Abramson, The Aloha system-another alternative for computer communications, in Proc 1970 Fall Joint Comput Conf AFIPS Conf. (Montvale, NJ. AFIPS Press, 1970), pp. 281-285

2. G Mergen, L Tong, Maximum asymptotic stable throughput of opportunistic slotted ALOHA and applications to CDMA networks. IEEE Trans Wireless Commun. 6, 1159-1163 (2007)

3. V Naware, G Mergen, L Tong, Stability and delay of finite-user slotted ALOHA with multipacket reception. IEEE Trans Inf Theory 51, 2636-2656 (2005). doi:10.1109/TIT.2005.850060

4. A Jamalipour, M Katayama, T Yamazato, A Ogawa, Transmit permission control on spread ALOHA packets in LEO satellite systems. IEEE J Sel Areas Commmun. 14, 1748-1757 (1996). doi:10.1109/49.545697

5. JJ Metzner, On improving utilization in ALOHA networks. IEEE Trans Commmun. 24, 447-448 (1976). doi:10.1109/TCOM.1976.1093317

6. YD Yao, AUH Sheikh, Outage probability analysis for microcell mobile radio systems with cochannel interferers in Rician/Rayleigh fading environment. Electron Lett. 26, 864-866 (1990). doi:10.1049/el:19900566
7. C van der Plas, JP Linnartz, Stability of mobile slotted AlOHA network with Rayleight fading, shadowing and near-far effects. IEEE Trans Veh Technol. 39, 359-366 (1990). doi:10.1109/25.61357

8. JP Linnartz, Near-far effects in land mobile random access networks with narrow-band Rayleigh fading channels. IEEE Trans Veh Technol. 41, 77-89 (1992). doi:10.1109/25.120148

9. J Ward, RT Compton Jr, Improving the performance of a slotted ALOHA packet radio ntwork with an adaptive array. IEEE Trans Commun. 40(2), 292-300 (1992). doi:10.1109/26.129191

10. J Ward, RT Compton Jr, High throughput slotted ALOHA packet radio networks with adaptive arrays. IEEE Trans Commun. 41(3), 460-470 (1993). doi:10.1109/26.221075

11. J Hsu, I Rubin, Performance analysis of directional random access scheme for multiple access mobile ad-hoc wireless networks, in Proc MILCOM. 1, 45-51 (2005)

12. L Zhou, Y Yao, H Heffes, Z Ruifeng, Investigation of slotted ALOHA under Nakagami fading with synchronized and asynchronous cochannel cells. IEEE Trans Veh Technol. 52(6), 1642-1651 (2003). doi:10.1109/TVT.2003.819622

13. M Yamada, Y Hara, Y Kamio, S Hara, Packet communications with slotted ALOHA in a mobile cellular system, in Proc VTC. 3, 1363-1367 (2001)

14. K Navaie, H Yanikomeroglu, Optimal downlink resoruce allocation for nonrealtime traffic cellular CDMA/TDMA networks. IEEE Commun Lett. 10(4), 278-280 (2006). doi:10.1109/LCOMM.2006.1613746

15. Y Zhu, Q Zhang, J Zhu, Improve transmission reliability with multi-AP diversity in wireless networks: architecture and performance analysis, in Proc. 3rd International Conference on Quality of Service in Heterogeneous Wired/Wireless Networks (2006)

16. R Rom, M Sidi, Multiple Access Protocols: Performance and Analysiss (Springer Verlag: New York, 1990)

17. J Arnbak, W Blitterswijk, Capacity of slotted ALOHA in Rayleigh-fading channels. IEEE J Sel Areas Commmun. 5, 685-692 (1987). doi:10.1109/ JSAC.1987.1146575

18. C Namislo, Analysis of mobile radio slotted ALOHA networks. IEEE Trans Veh Technol. 33, 199-204 (1984)

19. A Sheikh, Y Yao, X Wu, The ALOHA systems in shadowed mobile radio channels with slow or fast fading. IEEE Trans Veh Technol. 39(3), 289-298 (1990)

20. D Goodman, A Saleh, The near/far effect in local ALOHA radio communication. IEEE Trans Veh Technol. 36, 19-27 (1987)

doi:10.1186/1687-1499-2011-119

Cite this article as: Zheng and Yao: Slotted Aloha with multi-AP diversity and APS transmit beamforming. EURASIP Journal on Wireless Communications and Networking 2011 2011:119.

\section{Submit your manuscript to a SpringerOpen ${ }^{\circ}$ journal and benefit from:}

- Convenient online submission

- Rigorous peer review

- Immediate publication on acceptance

- Open access: articles freely available online

- High visibility within the field

- Retaining the copyright to your article

Submit your next manuscript at $\gg$ springeropen.com 